\title{
HEALTHCARE MONITORING SYSTEM USING ZIGBEE
}

\author{
Etukala Neeruganti Sai Kaushik ${ }^{1}$, Kumar Mani Chandra Y. M. ${ }^{2}$, Hem Bhupaal Reddy ${ }^{3}$, \\ Reena Kulkarni ${ }^{4}$ \\ ${ }^{1,2,3}$ Student, Department of Electronics \& Communication, BNM Institute of Technology, Karnataka, India \\ ${ }^{4}$ Assistant Professor, Department of Electronics \& Communication, BNM Institute of Technology, Karnataka, India
}

\begin{abstract}
Health care monitoring system is designed to monitor the health parameters of the patients andas an alarm system when the patients' health parameters undergo abnormal state. This system is designed such that it can effectively be used in hospitals as well as in homes. Using this device we can measure the most important health parameters like ECG, EEG, Heart Beat, Respiration and Body temperature. Even today ECG and EEG machines are very costly to employ them in our houses, keeping this in mind we have designed cost effective system. The use of wireless networks in the form of Zigbee allows this system to wirelessly monitor the health parameters in any computer and the integration of this project with G-mail helps in sending an alert to the registered Email address in the emergency situations.
\end{abstract}

Keywords:- ECG, EEG, Heart Beat, Respiration, Body Temperature,ZigBee, Alarm, E-mail ****

\section{INTRODUCTION}

With the advancement of the present day technology automation has been taking place enormously in every field. In order to minimize the hospital staff, burden on them, to operate the patients effectively and to measure health parameters by ourselves in homes, we have proposed this system.

In more populated countries, hospitals still suffers from effectively operating the patients as the number of patients is being operated with less hospital staff. The increase in patients has also led to the decrease in the relative number of doctors per patients which results in poor monitoring of a patient. To overcome all these problems automation and integration of different health measuring devices plays an important role.

Healthcare monitoring system is basically divided into three parts. (i) Sensing part, (ii) Monitoring part and (iii) Alarm part.

Under the sensing part the measurement of the five different health parameters takes place such as ECG, EEG, Heart Beat, Respiration and Body temperature. The techniques used for measuring of each parameter is discussed further. Under the monitoring part the measured parameters will be displayed on the LCD screen. The same health parameters are transmitted using zigbee and can be viewed in the PC connected to it. The ECG and EEG graphs are also displayed on PC. The use of G-mail in this system helps to send an alarm or warning mail to the emergency staff or to the doctor. The preference of choosing an e-mail over a message is discussed further. First we have to enter the threshold values for each health parameter. During measuring if any one of the health parameter records in non-threshold limits then immediately buzzer will start ringing as wells as an mail will be sent to the registered E-mail address using the PC's internet that can be from Wi-Fi or LAN or Dongle etc, all this comes under alarm part. The block diagram for the system is as showed below.

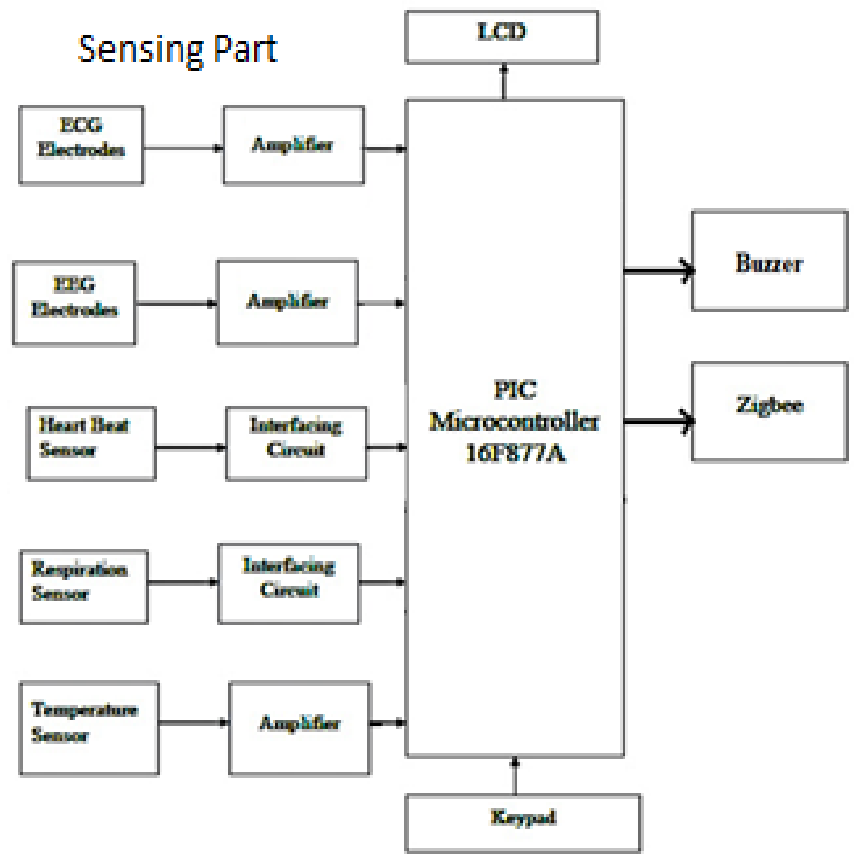

Monitoring Part

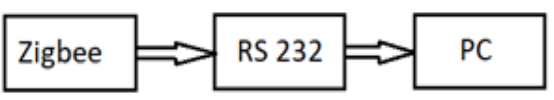

Alarm Part

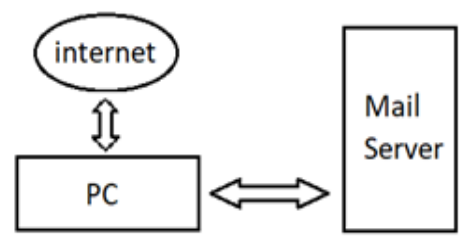




\subsection{ECG}

Electrocardiography is the process of recording the electrical activity of the heart over a period of time using electrodes placed on the skin. These electrodes detect the tiny electrical changes on the skin that arise from the heart muscle's electro physiologic pattern of depolarizing and repolarizing during each heartbeat. It is a very commonly performed cardiology test.

In a conventional 12-lead ECG, 12 electrodes are placed on the patient's limbs and on the surface of the chest. The overall magnitude of the heart's electrical potential is then measured from 12 different angles ("leads") and is recorded over a period of time (usually 10 seconds). In this way, the overall magnitude and direction of the heart's electrical depolarization is captured at each moment throughout the cardiac cycle. The graph of voltage versus time produced by this noninvasive medical procedure is referred to as an electrocardiogram.

The following fig is the circuit diagram for the ECG module we are using. Here we are using three electrode the first two has to be connected to two hands and third one to leg. The skin generate minute electric pulse during the heart activity. The electric pulses from the electrode 1 and electrode 2 are given to the instrumentation amplifier which gives the amplified output at the voltage follower, the minute signal will be amplified such that ir can be processed further, at filter circuit the noise will be eliminated. The noise can be generated due to motion of hands or due to surroundings etc, all these will be removed, the analog signal now will be converted into pulses at PWM and the digital values will be displayed on the lcd screen and these values will be transmitted to pc using zigbee. Graph will be drawn using the digital values provided.

\subsection{EEG}

Electroencephalography (EEG) is an electrophysiological monitoring method to record electrical activity of the brain. The signal acquisition techniques for the EEG is also same of ECG but the difference is the EEG is determined by the frequency in terms hertzs. so we are using the same module as of ECG to sense the signal. Once the signal is acquired, its frequency is determined by calculating the number of pulses generated by the brain in ten seconds. In general the accuracy is based upon the number of electrodes used to detect.

\subsection{Heart Beat}

Traditional models uses a set of electrode leads attached to the chest to determine the heart beat and there are some other techniques like chest straps to measure the heartbeat. In this system we are employing optical technique to measure the heartbeat. For that we are using heart beat sensor which works on IR rays.

The sensor unit consists of an infrared light-emitting-diode (IR LED) and a photodiode. The IR diode transmits an infrared light into the fingertip (placed over the sensor unit), and the photodiode senses the portion of the light that is reflected back. The intensity of reflected light depends upon the blood volume inside the fingertip. So, each heart beat slightly alters the amount of reflected infrared light that can be detected by the photodiode. With a proper signal conditioning, this little change in the amplitude of the reflected light can be converted into a pulse. The pulses can be later counted by the microcontroller to determine the heart rate.

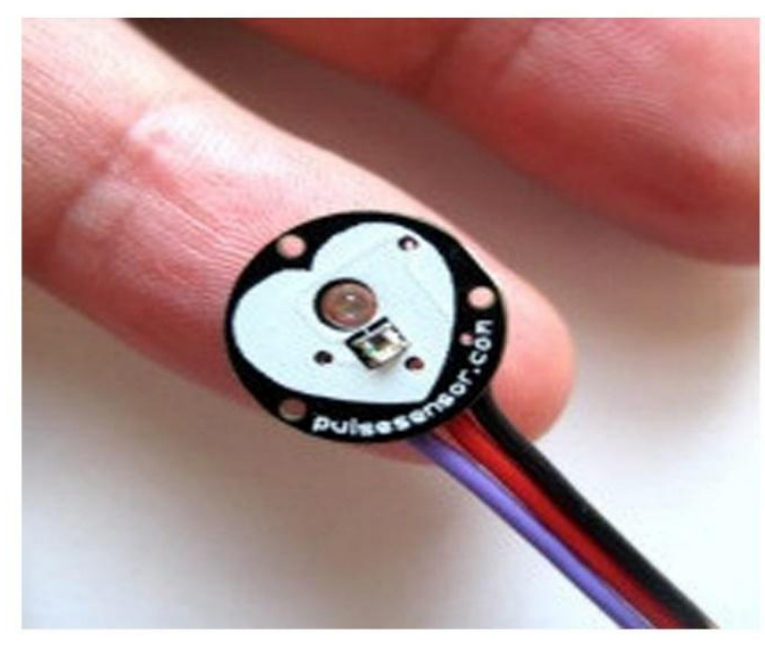

\subsection{Respiration}

In traditional techniques a chest band is used to determine the variations and based upon that respiration rate is measured. But that technique is not comfortable for patients since they have to wear the belt across their chest all the time, so we have implemented this in an easier way. Here we are using the fact that exhaling air from the body is always warmer than the inhaling air. So we are using two thermistors one is placed to determine the outside temperature and the other is placed near the nose or fixed to oxygen mask. The micro controller constantly compares the temperature of the both thermistors if it detects any difference then it means that air is exhaled. By counting the number of times exhaled for ten seconds the count for one minute is given.

\subsection{Temperature}

Here we are using a thermistor to measure the body temperature. When we place the figure on the thermistor the air film around it attain the temperature of the body. Thermistors works on the principle of the resistance decreases as temperature increases. Based on the voltage we get at the output temperature is predicted.

\subsection{Keypad}

Here five button keypad is used to enter the threshold values. Two for navigation front and back and two keys for increasing and decreasing and other for Enter. For example if the threshold value for the heart beat is set as $100 \mathrm{bpm}$ using keyboard. If the measured values is more than 100 immediately a mail will be sent and buzzer will be activated. 


\subsection{ZigBee}

We are using ZigBee for the communication between the system and PC, we preferred ZigBee over Bluetooth and WiFi for the fact that that the range and the speed of the ZigBee is more compared to that of Bluetooth. And the ZigBee is power efficient compared to $\mathrm{Wi}-\mathrm{Fi}$ and exposing to zigbee is less hazardous than exposing to $\mathrm{Wi}-\mathrm{Fi}$ for the patients. Here are we using XBP24CZWIT-004REVC version of ZigBee. It works at $2.4 \mathrm{GHz}$ frequency and have bandwidth of 250 kbps. At the receiving end pc is connected to ZigBee using RS232 cable. The ZigBee is configured in this system to send the data every ten seconds.

\section{SOFTWARE PART}

In the gui all the values of health parameters transmitted from the system is displayed along with whether the parameter in normal or abnormal condition. The waveforms for the ECG and EEG also displayed as shown in the figure. In the first column of the software we have to give the patients email address along with the password of it and the recipient doctors email address to send the mail.

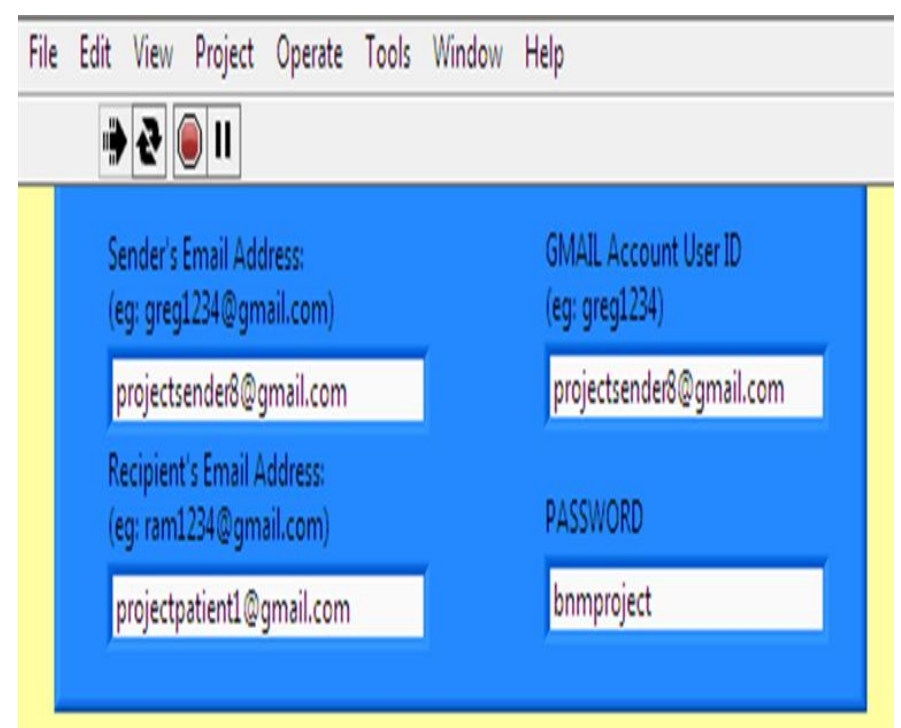

\section{CONFIGURING G-MAIL}

As G-mail is most commonly used email we are using Gmail in order to send mail address. Using this software we can use other e mails also but here as we are using Gmail we are going to explain how to configure G-mail. After giving the mail address g-mail is not going to send the mail it has to configure certain setting. First we have to log in to Gmail and then click on profile picture and then on my account and then click on security and sign in and then scroll down and you can see an option stating allow less secure apps and that should be turned on. Now we can send the mails with our system. All the configurations and usage of the g-mail is under the license and agreements and no violations of rules and agreements are made.

\section{PROTOTYPE}

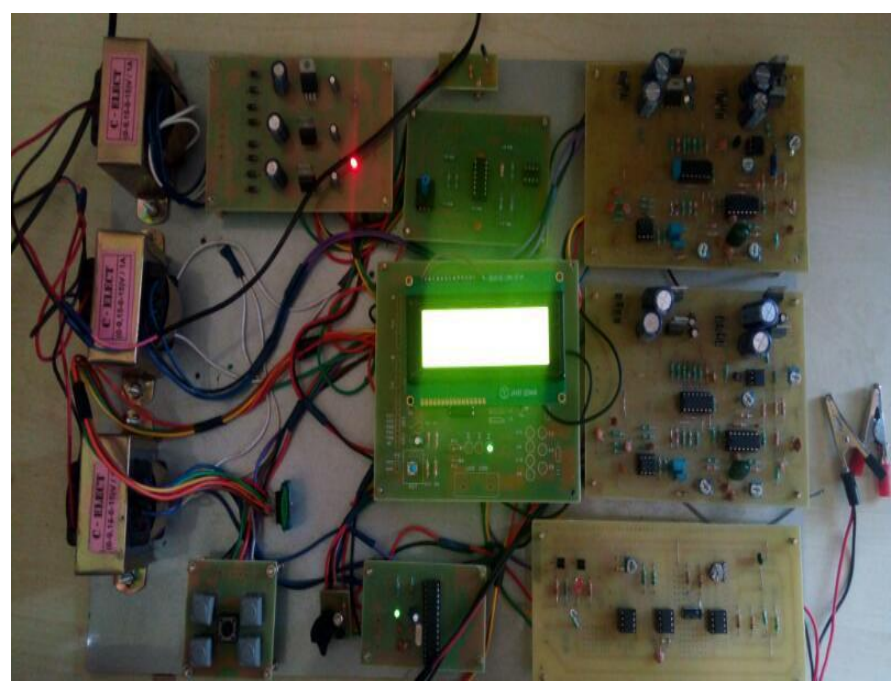

\section{FLOW CHART}

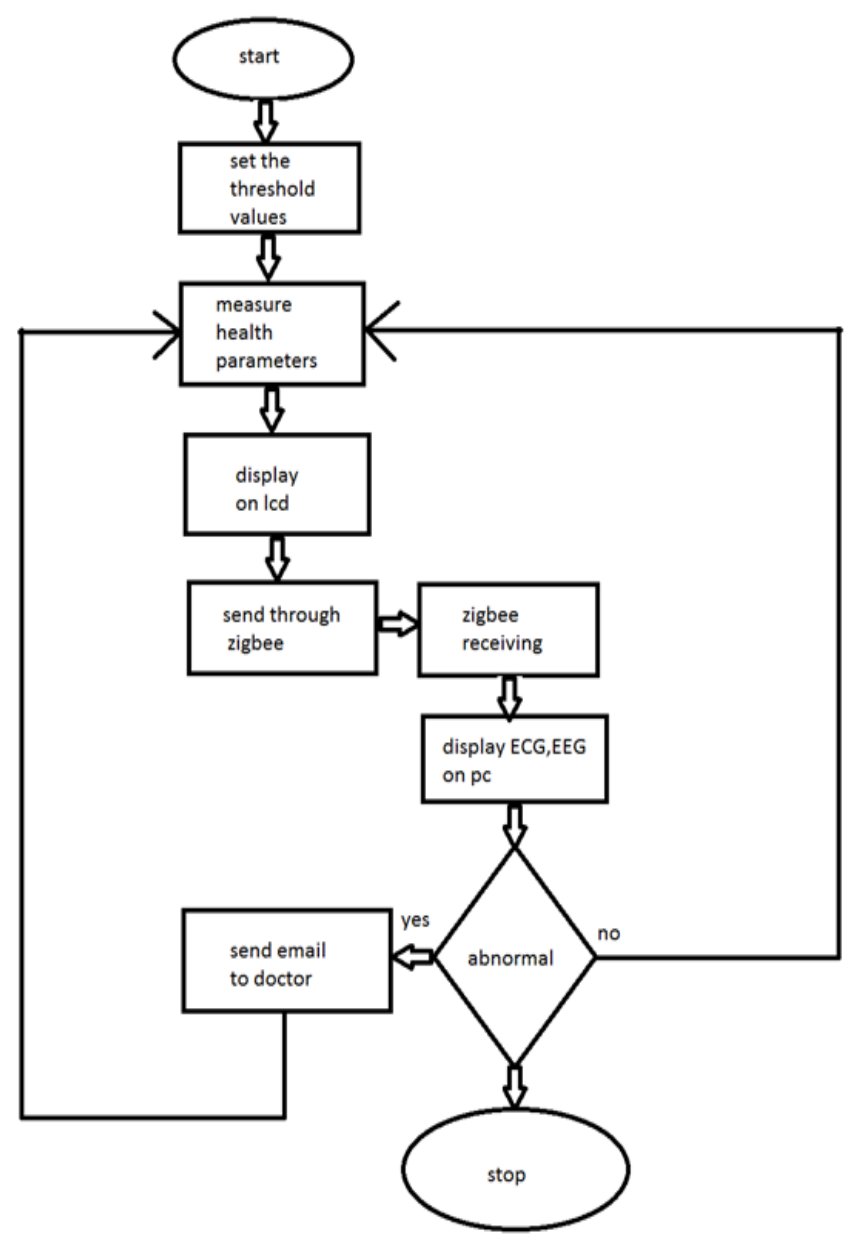

\section{RESULT}

All the different health parameters are being displayed in the LCD as shown in the figure. The same parameters in are being showed in PC along with the ECG and EEG waveforms. The mails received from the patients are being showed in the figure. 


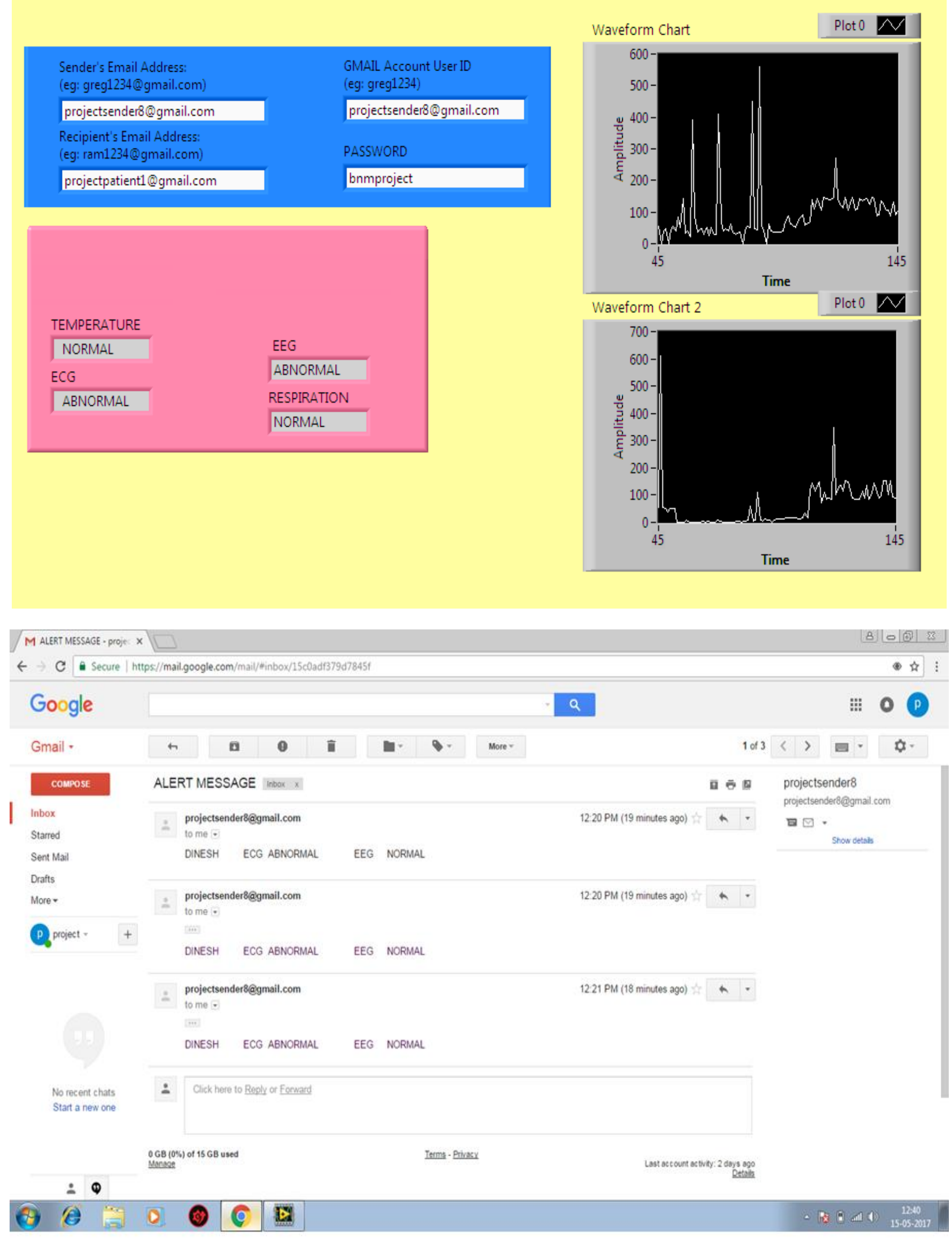




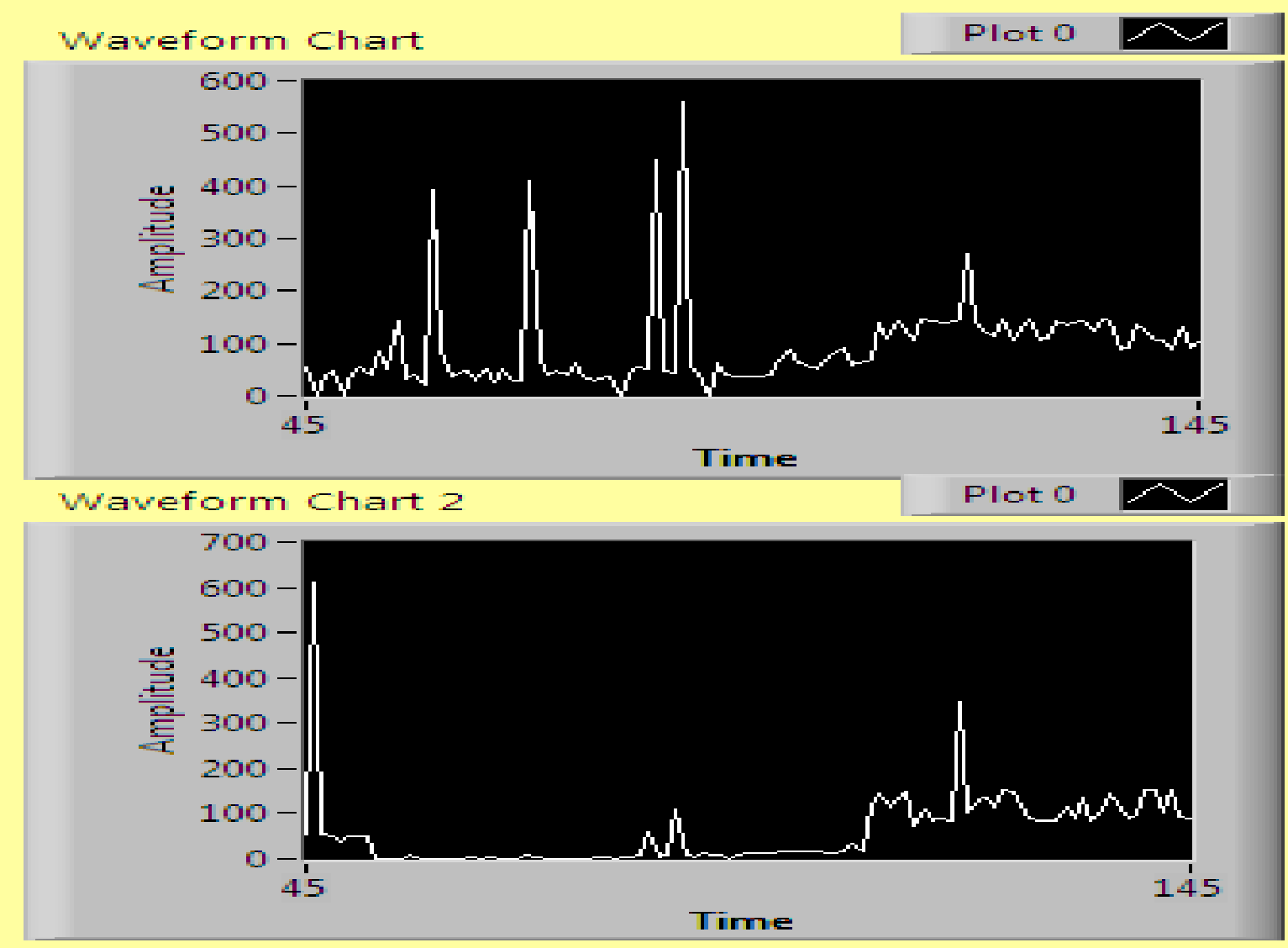

\section{CONCLUSION}

The system is implemented with low cost and it can be used effectively in homes and hospitals. Although some parameters'accuracy is not high, the accuracy can be increased using more electrodes in case of ECG and EEG. In further this system can be developed by integrating this with the android apps for monitoring and usage of internet for communication over long ranges.

\section{REFERENCES}

[1]. Salyer J. 2003. Neonatal and Pediatric Pulse Oximetry. RespiratoryCare 286-289.

[2]. C.Otto,Milenkovic,A.,Sanders,C., Jovanov,E.,"System architecture ofa wireless body area sensor network for ubiquitous healthmonitoring," Journal of Mobile Multimedia, vol. 1, pp. 307-326,2006

[3]. Naazneen M. G. et al, "Design and Implementation ofECG Monitoring and Heart Rate Measurement System",International Journal of Engineering Science andInnovative Technology (IJESIT), vol. 2, Issue 3, pp.456465, May 2013.

[4]. EmimalJabason; JudesMonisha R A, "Single Channel Real-Time Wireless ECG Monitoring System” Sep-2014 [5]. Samson Nivins. S "Application of Single Channel Wireless EEG -Detection of Alzheimer's Disease" by, Apr2014

[6]. Mr. Aniket V. Kale,Miss. Supriya D. Gawade, Miss. Sayali Y. Jadhav and Mr. Samrat A. Patil "GSM Based Heart Rate and Temperature Monitoring System" may 2015
[7]. Jigar Chauhan, SachinBojewar" Sensor Networks based Healthcare Monitoring System” Apr-2014

[8]. Sakshisharma, RashmiVashisth, " Zigbee Based Centralized Patient Monitoring System",2015 International Conference on Computational and Communication Networks (pp.212 - 217)

[9]. Hongzhou YU, Lu Liu, "Remote Health Monitoring System Using Zigbee Network and GPRS Transmission Technology" 2011 Fourth International Symposium on Computational Intelligence and Design (pp 151 - 154 ) 